\title{
A Word to the WISE: Confusion is Unavoidable for WISE-selected Infrared Excesses
}

\author{
Erik Dennihy ${ }^{1}(\mathbb{B})$ Jay Farihi $^{2}$ (i), Nicola Pietro Gentile Fusillo ${ }^{3,4}$ (1) , and John H. Debes ${ }^{5}$ (1) \\ ${ }^{1}$ NSF's National Optical-Infrared Astronomy Research Laboratory, Gemini Observatory, Colina el Pino S/N, La Serena, Chile; edennihy@gemini.edu \\ ${ }^{2}$ Department of Physics and Astronomy, University College London, London WC1E 6BT, UK \\ ${ }^{3}$ Department of Physics, University of Warwick, Coventry, CV4 7AL, UK \\ ${ }^{4}$ European Southern Observatory, Karl-Schwarzschild-Str 2, D-85748 Garching, Germany \\ ${ }^{5}$ ESA for AURA, Space Telescope Science Institute, 3700 San Martin Drive, Baltimore, MD 21218, USA \\ Received 2019 December 19; revised 2020 January 20; accepted 2020 January 22; published 2020 March 9
}

\begin{abstract}
Stars with excess infrared radiation from circumstellar dust are invaluable for studies of exoplanetary systems, informing our understanding of processes of planet formation and destruction alike. All-sky photometric surveys have made the identification of dusty infrared excess candidates trivial, however, samples that rely on data from Wise Infrared Survey Explorer (WISE) are plagued with source confusion, leading to high false-positive rates. Techniques to limit its contribution to WISE-selected samples have been developed, and their effectiveness is even more important as we near the end-of-life of Spitzer, the only facility capable of confirming the excess. Here, we present a Spitzer follow-up of a sample of 22 WISE-selected infrared excess candidates near the faint-end of the WISE detection limits. Eight of the 22 excesses are deemed the result of source confusion, with the remaining candidates all confirmed by the Spitzer data. We consider the efficacy of ground-based near-infrared imaging and astrometric filtering of samples to limit confusion among the sample. We find that both techniques are worthwhile for vetting candidates, but fail to identify all of the confused excesses, indicating that they cannot be used to confirm WISE-selected infrared excess candidates, but only to rule them out. This result confirms the expectation that WISE-selected infrared excess samples will always suffer from appreciable levels of contamination, and that care should be taken in their interpretation regardless of the filters applied.
\end{abstract}

Unified Astronomy Thesaurus concepts: White dwarf stars (1799); Circumstellar dust (236); Infrared excess (788)

\section{Introduction}

In the era of database astronomy, the construction of spectral energy distributions (SEDs) from the ultra-violet to the midinfrared for large samples of stars is straightforward, requiring little user-input or effort. Modern tools such as the VO SED Analyzer (Bayo \& Rodrigo 2008) can even detect infrared excesses for thousands of candidates at a time in a completely automated fashion. Many subfields have benefited from the ease-of-use of catalog photometry, though they are not without pitfalls. Searches for infrared excesses from warm (1000 K), circumstellar dust provide a good case-study of the benefits and drawbacks of analyzing SEDs using only catalog photometry.

Circumstellar dust is a signpost for planetary systems, indicating the on-going process of planetary formation around pre-main and main-sequence stars (Kennedy \& Wyatt 2012; Patel et al. 2014; Cotten \& Song 2016; Binks \& Jeffries 2017), and illuminating the post-main-sequence destruction of remnant planetary systems around white dwarf stars (Debes et al. 2011b; Hoard et al. 2013; Barber et al. 2014; Dennihy et al. 2017). The frequency of circumstellar dust around stellar sources informs planetary occurrence rates in instances where direct detection is not feasible. These searches rely heavily on data from the Wise Infrared Survey Explorer (WISE; Wright et al. 2010), which produced the only all-sky survey at the wavelengths where warm dust is most apparent $(\lambda \geqslant 3 \mu \mathrm{m})$. But the coarse spatial resolution of WISE leads to a high probability of source confusion, contaminating samples of WISE-selected infrared excess with false positives and skewing statistical studies of warm dust frequency.

Estimates of contamination by source confusion for WISEselected dusty infrared excesses around main-sequence stars indicate false-positive rates as high as 70\% (Silverberg et al. 2018).
Dusty infrared excesses around white dwarf stars are much fainter than their main-sequence counterparts, and typically only detected in $W 1$ and $W 2$ bands (see Farihi 2016 for a recent review). Their faint magnitudes push the boundaries of the source confusion limited detection thresholds of the AllWISE surveys. More concerning, as the Spitzer Space Telescope (Werner et al. 2004) reaches its end-of-life, the ability to confirm WISE infrared excesses for large samples may be lost entirely. The effectiveness of the next generation observatory, the James Webb Space Telescope (JWST) (Gardner et al. 2006), to mimic the survey imaging capability of Spitzer will be limited by initial slew times that are an order of magnitude larger. This is likely to mean JWST cannot support this science effectively for large samples of dusty white dwarfs as are currently being identified with Gaia (RebassaMansergas et al. 2019).

In this paper, we present Spitzer follow-up of a sample of 22 WISE-selected infrared excess candidates around white dwarf stars and discuss the efficacy of techniques to limit the contamination of WISE-selected infrared excesses by source confusion. This sample approaches the faint limit of the AllWISE surveys, making it of broader impact to studies of source confusion among WISE-selected infrared excesses. Using the higher-resolution Spitzer data, we confirm the WISE infrared excesses in 14/22 systems, with the remaining systems all showing nearby sources within the WISE beam.

Prior to their Spitzer observations, all of our targets were vetted by examining ground-based near-infrared imaging and astrometric shifts to probe for clear instances of confused WISE photometry. None of the eight contaminated systems showed nearby sources in their ground-based near-infrared imaging, demonstrating that it is insufficient to rule out source confusion at the WISE bands. We find that the astrometric information is a 
Table 1

Spitzer and WISE Fluxes for Each Candidate, Separated Into Spitzer Confirmed Excesses and Confused WISE Photometry

\begin{tabular}{|c|c|c|c|c|c|c|c|c|}
\hline Target Name & Gaia WD Designation $^{\mathrm{a}}$ & \multicolumn{2}{|c|}{ Spitzer } & \multicolumn{2}{|c|}{ WISE } & $\begin{array}{l}\mathrm{S} / \mathrm{N} \\
(W 1)\end{array}$ & \multicolumn{2}{|c|}{ Gaia } \\
\hline ATLAS 00254 & WD J002540.01-393454.56 & $39 \pm 3$ & $42 \pm 3$ & $34 \pm 5$ & $33 \pm 9$ & 7.3 & 5.5 & 0.66 \\
\hline ATLAS 02325 & WD J023252.01-095745.86 & $49 \pm 3$ & $40 \pm 3$ & $48 \pm 5$ & $41 \pm 10$ & 10.8 & 7.1 & 0.19 \\
\hline ATLAS 10552 & WD J105524.50-023721.13 & $86 \pm 5$ & $86 \pm 5$ & $104 \pm 7$ & $98 \pm 13$ & 16.4 & 0.2 & 0.83 \\
\hline ATLAS 22120 & WD J221202.88-135239.96 & $156 \pm 8$ & $156 \pm 8$ & $132 \pm 7$ & $145 \pm 13$ & 19.3 & 8.7 & 0.07 \\
\hline ATLAS 23403 & WD J234036.64-370844.72 & $169 \pm 9$ & $161 \pm 9$ & $155 \pm 7$ & $158 \pm 11$ & 24.4 & 9.4 & 0.05 \\
\hline EC 01071 & WD J010933.16-190117.56 & $95 \pm 6$ & $79 \pm 5$ & $89 \pm 6$ & $99 \pm 11$ & 15.7 & 5.6 & 0.46 \\
\hline EC 01129 & WD J011501.17-520744.67 & $55 \pm 4$ & $34 \pm 2$ & $71 \pm 6$ & $27 \pm 9$ & 14.2 & 6.6 & 0.29 \\
\hline EC $21548^{\mathrm{b}}$ & WD J215823.88-585353.81 & $199 \pm 11$ & $151 \pm 8$ & $205 \pm 8$ & $171 \pm 10$ & 29.1 & $\cdots$ & $\cdots$ \\
\hline SDSS 01190 & WD J011909.99+104454.09 & $89 \pm 5$ & $87 \pm 5$ & $90 \pm 6$ & $92 \pm 11$ & 16.0 & 7.5 & 0.22 \\
\hline \multicolumn{9}{|c|}{ Confused WISE Photometry } \\
\hline ATLAS 22561 & WD J225612.92-131938.83 & $91 \pm 5$ & $60 \pm 4$ & $119 \pm 7$ & $74 \pm 13$ & 17.1 & 0.4 & 0.72 \\
\hline EC 02566 & WD J025859.58-175020.33 & $40 \pm 3$ & $22 \pm 2$ & $48 \pm 5$ & $56 \pm 8$ & 11.9 & 3.4 & 0.77 \\
\hline EC 03103 & WD J031121.31-621515.72 & $81 \pm 5$ & $53 \pm 3$ & $157 \pm 6$ & $128 \pm 8$ & 30.4 & 0.0 & 0.42 \\
\hline EC 05276 & WD J052912.10-430334.49 & $71 \pm 4$ & $41 \pm 3$ & $112 \pm 5$ & $65 \pm 8$ & 23.3 & 3.0 & 0.45 \\
\hline SDSS 00021 & WD J000216.18+073350.30 & $30 \pm 3$ & $19 \pm 2$ & $40 \pm 6$ & $37 \pm 12$ & 7.7 & 5.6 & 0.55 \\
\hline SDSS $08304^{c}$ & WD J083047.28+001041.51 & $28 \pm 3$ & $27 \pm 2$ & $26 \pm 6$ & $35 \pm 11$ & 5.0 & 0.3 & 2.03 \\
\hline SDSS 13054 & WD J130542.73+152541.16 & $37 \pm 3$ & $23 \pm 2$ & $80 \pm 6$ & $57 \pm 12$ & 14.7 & 5.0 & 0.52 \\
\hline SDSS 13570 & WD J135701.68+123145.62 & $9 \pm 2$ & $6 \pm 1$ & $21 \pm 5$ & $18 \pm 9$ & 5.2 & 4.5 & 0.92 \\
\hline
\end{tabular}

Notes. In the final two columns, we present the Gaia Figure of Merit (FoM) and separation from expected position collected from the official Gaia-AllWISE crossmatch, discussed in Section 3.2.

${ }^{a}$ Gentile Fusillo et al. (2019).

b The Gaia-AllWISE cross-match returned no results for EC 21548, despite an AllWISE detection within 0". 5 of the expected position. This case is discussed in Section 3.2 .

${ }^{\mathrm{c}}$ The measured IRAC-Ch 1 and $\mathrm{Ch} 2$ fluxes are confused with a background galaxy.

more useful indicator of the potential for source confusion, but only when considering the full astrometric uncertainty of the surveys involved. Even when applied carefully, we demonstrate that these techniques will not result in a clean sample of excesses, and studies based on WISE-selected infrared excesses should always consider a level contamination when interpreting sample properties.

\section{Spitzer View of WISE Infrared Excess Candidates}

Our targets were selected from a handful of studies that applied different criteria to identify the infrared excesses (Dennihy et al. 2017, N. P. Gentile Fusillo et al. 2020, in preparation). The common property of our targets is an infrared excess in the WISE W1 and W2 consistent with a warm, compact dust disk around a white dwarf star. The Spitzer photometry is superior to the WISE photometry in both sensitivity and, more importantly, spatial resolution, allowing us to test the possibility that a given WISE excess is the result of source confusion. For each target, we searched for instances of multiple sources within the WISE beam, and compared the Spitzer photometry against stellar models to confirm the WISEselected excess.

\subsection{IRAC Imaging and Photometry}

Under program 14100, we collected 3.6 and $4.5 \mu \mathrm{m}$ photometry of 22 dusty white dwarf candidates using the Infrared Array Camera (IRAC; Fazio et al. 2004) with Spitzer in Cycle 14. Ten frames were taken using $30 \mathrm{~s}$ exposures with the medium-sized cycling dither pattern, resulting in $300 \mathrm{~s}$ of total integration in each channel. We produced fully calibrated mosaic images for each target using the MOPEX software package (Makovoz et al. 2006) following the recipes outlined for point-source extraction in the Spitzer Data Analysis Cookbook version 6.0. point-spread function-fitted photometry was conducted using APEX, and the error in the measured flux was summed in quadrature with a $5 \%$ calibration uncertainty (Farihi et al. 2008). It has been demonstrated that well-dithered observations are robust against intra-pixel flux variations at the sub-percent level (Wilson et al. 2019) so we did not apply any such corrections. The measured fluxes are presented in Table 1.

For each target, we examined the IRAC-Ch 1 and $\mathrm{Ch} 2$ mosaic images for multiple sources within the WISE beam, centered on the AllWISE detection. The critical distance for resolving neighboring sources is $1.3 \times$ the FWHM of the pointspread function of a given band ( $7 !$ ! 8 for Wl). Within this separation, the AllWISE pipeline relies on an active deblending 

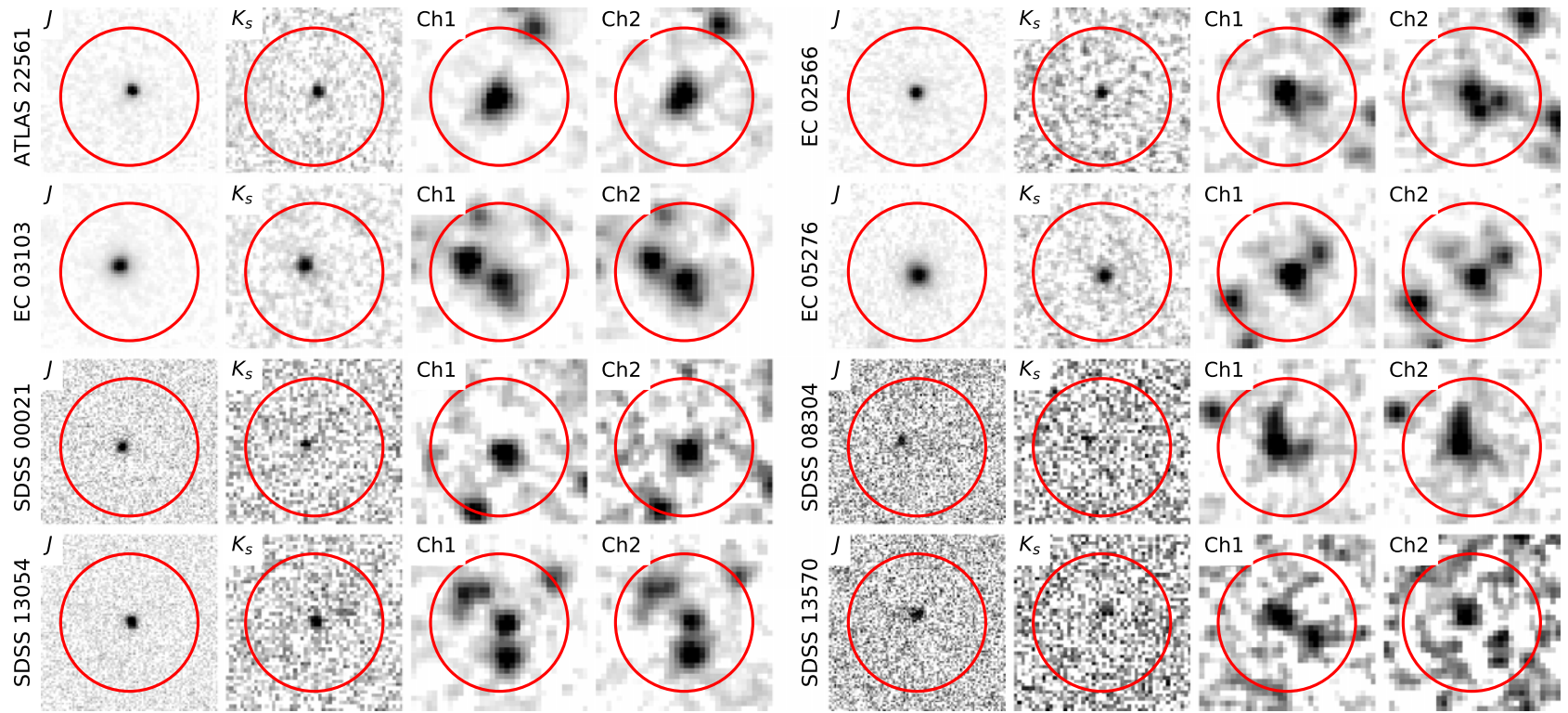

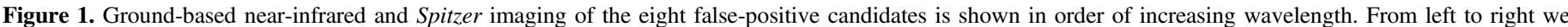

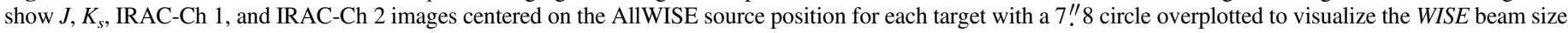

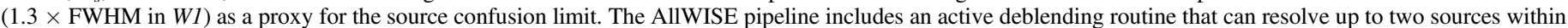

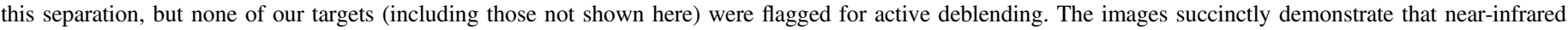
imaging is insufficient to rule out source confusion in the WISE W1 and W2 bands.

procedure to detect instances of source confusion, triggered by an unsatisfactory fit to the intensity distribution during the point-source fitting photometry routine. ${ }^{6}$ None of our targets were flagged for the active deblending routine so we adopted a $7 !$. 8 radius as our limit for potential source confusion.

Eight of our 22 targets have multiple sources within this limit indicating the AllWISE photometry was potentially confused. In Figure 1 , we show $10^{\prime \prime} \times 10^{\prime \prime}$ cutouts of the publicly available near-infrared $J$ and $K_{s}$ and IRAC-Ch 1 and Ch 2 images of these eight targets. We note that in all eight, the nearby contaminants are not detected in any of the near-infrared images. We discuss the efficacy of near-infrared imaging for limiting contamination in WISE-selected samples in Section 3.1.

\subsection{Comparison with Stellar Models}

We constructed SEDs for each target utilizing data from the Galaxy Evolution Explorer (Martin et al. 2005), Sloan Digital Sky Survey (SDSS; Ahn et al. 2014), VST-ATLAS survey (Shanks et al. 2015), Panoramic Survey Telescope and Rapid Response System (Chambers et al. 2016), SkyMapper Southern Survey (Wolf et al. 2018), UKIRT Infrared Deep Sky Survey (Lawrence et al. 2007), VISTA Hemisphere Survey (VHS; Irwin et al. 2004; Hambly et al. 2008; Cross et al. 2012), and AllWISE surveys (Cutri et al. 2013b). We dereddened the photometry using a standard prescription (Gentile Fusillo et al. 2019) and converted the magnitudes into fluxes using the published zero-points for each bandpass.

Most of the objects in our sample do not have a published spectrum to help us choose an appropriate stellar model, instead they have only been classified as white dwarf stars. The "EC" objects were first identified with low-resolution spectrograms as part of the Edinburgh-Cape Blue Object Survey (Stobie et al. 1997), and later confirmed with targeted followup (Dennihy et al. 2017). The "ATLAS" and "SDSS" objects

\footnotetext{
6 http://wise2.ipac.caltech.edu/docs/release/allsky/expsup/sec4_4c.html
}

were identified as high probability white dwarf candidates via their photometry and proper motion (Girven et al. 2011; Fusillo et al. 2015; Gentile Fusillo et al. 2017). All of our objects were also included in the Gaia white dwarf catalog of Gentile Fusillo et al. (2019), which includes estimates of effective temperature and surface gravity assuming both hydrogen and helium dominated atmospheres.

For our stellar models, we utilized the pure hydrogendominated white dwarf model spectra of Koester (2010), with the effective temperature and surface gravity of each star taken from the hydrogen model fits to the Gaia photometry (Gentile Fusillo et al. 2019). It should be emphasized that in our comparison of the model to the SED, the model parameters were not being refit to the photometry, rather the surface gravity and effective temperature were fixed and the model was then scaled to fit the optical photometry. Because the goal of this exercise was only to identify the systems with an infrared excess, rather than to fit or describe the infrared excess, this approach was sufficient.

We determined the flux excess of each target in the IRACCh 1 and $\mathrm{Ch} 2$ bands using the standard formula:

$$
\chi=\frac{F_{\mathrm{obs}}-F_{\mathrm{mod}}}{\sqrt{\sigma_{\mathrm{obs}}^{2}+\sigma_{\mathrm{mod}}^{2}}}
$$

and deemed those that have a $\mathrm{Ch} 1$ or $\mathrm{Ch} 2$ flux excess greater than $4 \sigma$ and clean IRAC-Ch 1 and Ch 2 images to be Spitzer confirmed excesses. Targets that showed IRAC photometry consistent with the stellar model and had multiple sources within our 7!! 8 confusion limit were the result of confused WISE photometry. We present an example SED in Figure 2 that shows an instance of a contaminated excess produced by source confusion. The contaminating sources for this target, EC 03103, are clearly identifiable in Figure 1. The remainder of the SEDs are shown in Appendix and in Table 1, we identify the remaining cases of confused WISE photometry. 


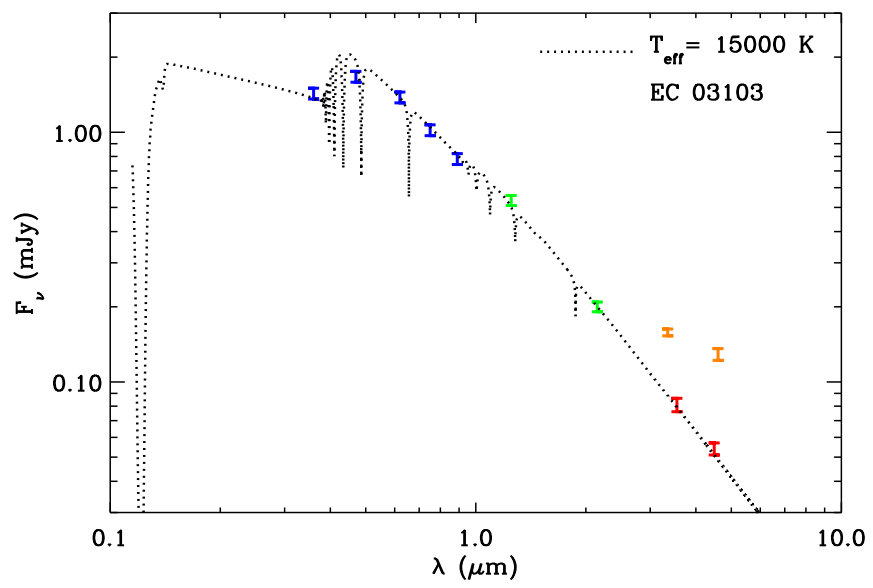

Figure 2. SED of EC 03103 demonstrates a case of confused WISE photometry (orange) erroneously being classified as an excess. The Spitzer photometry of the white dwarf (red) is consistent with the stellar model, and the confused sources that produced the WISE excess are clearly resolved with Spitzer in Figure 1. The remainder of the SEDs are shown in the Appendix.

Among our sample of 22 targets, we identify eight WISEselected excesses that are the result of source confusion, for a nominal contamination rate of $36 \%$. It is worth reemphasizing that our sample had already been vetted for obvious cases of source confusion prior to observation with Spitzer, so this $36 \%$ contamination rate only includes cases of source confusion that were unable to rule out with ground-based data. The effectiveness of these vetting techniques is discussed below.

\section{Mitigating Contamination in WISE-selected Samples}

As Spitzer nears the end of its operational lifetime, it is worth considering what techniques are effective at separating the clean from the confused among WISE-selected infrared excess samples. Recent works have explored this subject using samples of main-sequence stars (Patel et al. 2017; Silverberg et al. 2018), but the infrared excesses exhibited by dusty debris around white dwarf stars are much fainter, and typically only detected in the $W 1$ and $W 2$ bands. Furthermore white dwarf infrared excess searches are often limited to a few dozen candidates, so statistical methods for isolating outliers (such as demonstrated by Patel et al. 2017) are untenable. In the following sections, we consider a few commonly employed strategies and discuss their effectiveness based our classifications with Spitzer.

\subsection{Ground-based Near-infrared Imaging}

In the absence of space-based follow-up, ground-based nearinfrared imaging can be used to search for instances of multiple sources within the WISE imaging beam. The Two Micron All Sky Survey (Skrutskie et al. 2006) is insufficient in both depth and resolution for these purposes. The UKIDSS Large Area Survey (Lawrence et al. 2007) and the VISTA-VHS (McMahon et al. 2013) have depths of $K \approx 18.2 \mathrm{mag}$ and $K_{s} \approx 19.8$ mag, and their images have proven useful for quantifying levels of source confusion (e.g., Debes et al. 2011a; Dennihy et al. 2016). In the absence of publicly available imaging, targeted programs can also be used to cull samples of WISE-selected infrared excesses (Barber et al. 2012). Near-infrared imaging is preferred to optical in order to get as close as possible to the bandpass of WISE images. Ultracool dwarfs only become apparent beyond $1 \mu \mathrm{m}$ (Baraffe et al. 2015) and dusty background galaxies can rise in flux as a power law at the WISE wavelengths, escaping detection at optical and even nearinfrared wavelengths.

Prior to their selection for follow-up with Spitzer, all 22 of our targets were vetted for nearby sources within the WISE beam using high-quality, ground-based near-infrared images. In Figure 1, we show the $J$ - and $K_{s}$-band images for the eight contaminated targets. It is apparent from these image sequences that a clean near-infrared image is insufficient to confirm a WISE-selected infrared excess candidate. Near-infrared imaging is, however, a valuable tool for ruling out WISE-selected infrared excess candidates in cases where a clear, nearby source can be identified. It should always be considered for vetting candidates when available.

\subsection{Astrometric Separation}

Another method to assess the potential for source confusion of a WISE-selected infrared excess is to compare its expected position to the detected AllWISE detection. A sufficiently bright and nearby contaminant can be expected to shift the centroid of the detected source in the WISE images, indicating source confusion (Wilson \& Naylor 2017, 2018).

Prior to their Spitzer observations, our candidates were also vetted for large separations between their expected, proper motion-corrected Gaia position and their detected AllWISE position. All but one candidate was found within $1^{\prime \prime}$ of its proper motion-corrected position. The contamination rate in our sample indicates that at the subarcsecond level, the raw separation value between the expected and detected positions is a poor indicator of source confusion. This can be seen by comparing the separations in Table 1, where there is a large scatter and overlap between the confirmed and confused samples. The cause of this scatter is the wide range of WISE astrometric uncertainty among our targets, and is a by-product of our sample being near the fainter end of the AllWISE detection limits. Incorporating this astrometric uncertainty is essential for discriminating clean and confused WISE photometry, as discussed below.

\subsection{The Gaia FoM As a Confusion Discriminant}

The astrometric uncertainty of WISE is known to be inversely proportional to the detection's signal-to-noise $(\mathrm{S} / \mathrm{N})$. For the $W 1$ band, this relationship can be approximated as 3.0/(S/N) (Debes et al. 2011b; Cutri et al. 2013a). At the $5 \sigma$ detection limits of AllWISE, the astrometric uncertainty reaches $0 . .6$, meaning that in samples of a few hundred one reasonably expects several true detections of objects at separations greater than 0.5 . Conversely, and perhaps more detrimental, an object with high $\mathrm{S} / \mathrm{N}$ within a separation of 0 ." 5 could in fact be several standard deviations away from its expected position. Both cases emphasize that the raw separations should not be directly compared between bright and faint objects, and instead the individual astrometric uncertainty must be considered.

The framework developed for probability-based crossmatches provides a useful way to incorporate the astrometric uncertainty into the evaluation of whether or not the WISE astrometric position is likely perturbed (see Wilson \& Naylor 2018 for example). Additionally, the positional accuracy and proper motions provided by the Gaia Data Release 2 (Gaia Collaboration et al. 2016, 2018) provide a 


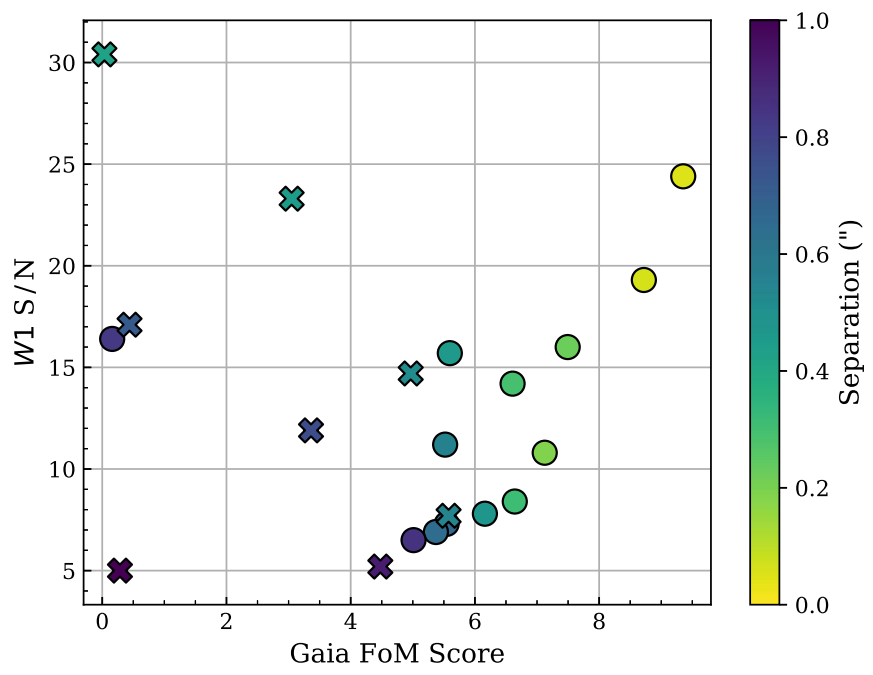

Figure 3. Gaia FoM is plotted against the AllWISE S/N for each object, with confirmed excesses shown as circles and excesses due to WISE source confusion as crosses. The color scale represents the separation between the expected position and the AllWISE detection. Candidates with a high W1 S/N but low FoM score are likely cases of confused AllWISE photometry.

fantastic reference position. As part of the Gaia DR2, crossmatched catalogs between several optical and near-infrared surveys were produced based on probabilistic, nearest-neighbor approaches (Marrese et al. 2017, 2019) that incorporate the astrometric uncertainty of each survey, the epoch differences between each catalog, and the probability of randomly finding a nearby, unrelated counterpart in a survey given the local source count density.

The cross-match algorithm works by first searching for all possible counterparts (dubbed neighbors) in a given catalog within $5 \sigma$ of the combined astrometric uncertainty of the object in Gaia and the neighbors in the catalog of interest. The FoM is computed for each potential neighbor by comparing the probabilities of discovery of the object at the measured separation and the probability of chance alignment. The counterpart with the highest FoM is selected as the match and reported in the bestNeighbor table (Marrese et al. 2017). All neighbors for each cross-match are listed in the corresponding Neighborhood table.

There is no threshold for the FoM score to use to evaluate the goodness of a match, that is to say the FoM does not translate directly into a likelihood. For the AllWISE catalog, this dimensionless parameter ranges from $7.0 \times 10^{-5}$ to 15.5 (Marrese et al. 2019), with a strong dependence on the astrometric uncertainty of the counterpart in AllWISE. As the AllWISE astrometry is inversely proportional to the $\mathrm{S} / \mathrm{N}$ ratio of the detection, one expects a relationship between the W1 S/N and the Gaia FoM score. We queried the Gaia Neighborhood catalog for and collected the recorded separation and FoM score of the best neighbor identified in the Gaia cross-match.

Figure 3 demonstrates a strong relationship between the Gaia FoM score and the W1 S/N, where the majority of the outliers are cases of confused AllWISE photometry. Based on this, we conclude that excesses with $\mathrm{S} / \mathrm{N}>10$ but FoM $<4$ are likely the result of source confusion. There is one object in this region, ATLAS 10552, that is a confirmed excess. A closer inspection of the images and SED for ATLAS 10552 indicate it is the rare case where the AllWISE photometry was confused in addition to the white dwarf having a true infrared excess as there is a faint, nearby source and the IRAC fluxes are slightly below the AllWISE fluxes.

Another object, EC 21548, returned no neighbors in the Gaia-AllWISE cross-match, i.e., there is not an associated source to the Gaia detection in the AllWISE catalog within $5 \sigma$ of astrometric separation. The AllWISE photometry we associated with EC 21548 corresponds to a source found at a separation of 0." 5 from the expected position of EC 21548. The Spitzer images show a single source near the expected position of EC 21548, and the IRAC-Ch 1 and Ch 2 fluxes agree with the AllWISE $W 1$ and $W 2$ fluxes, leading to a bit of mystery as to why the Gaia and the nearest AllWISE coordinates are so discrepant. Its exclusion in the cross-match could indicate some unaccounted for systematic uncertainty in the AllWISE astrometry, or it could simply be spurious. Whatever the case, it is another good example of a target that would have been erroneously rejected by the astrometric uncertainty cut proposed above.

In addition to the two confirmed excesses that would have been rejected, a few cases of confused WISE photometry are not distinguished by this method. SDSS 00021, SDSS 13054, and SDSS 13570 all lie near the sample of confirmed infrared excesses. The first is a case of a statistically weak infrared excess, and can be discarded for the purpose of evaluating this technique. Referencing the Spitzer images in Figure 1, we see that the remaining two have multiple sources contaminating the AllWISE photometry, resulting in a smaller positional perturbation than cases where one contaminant is responsible for the AllWISE positional offset.

In general, the Gaia FoM is a useful discriminant for identifying confused WISE photometry, having correctly identified five out of the eight confused sources in our sample. Applying this technique would have come at a cost though, as two confirmed excesses were rejected by this method and the two cases of multiple contaminants that result in little astrometric perturbation would have been missed. These results emphasize that even advanced astrometric methods will fail to produce clean samples of WISE-selected infrared excesses.

\subsection{Proper Motion Comparison}

Related to the astrometric test, one can also compare the proper motions measured by WISE and Gaia to test the validity of a WISE-selected infrared excess (Debes et al. 2019). This is effectively repeating the astrometric experiment with a series of independent measurements over time. Given the six month baseline, the initial WISE proper motions are not sufficient for comparison with Gaia, but the continued observations of the NEOWISE mission (Mainzer et al. 2014) have provided a six year baseline allowing for improved motion measurements. The CatWISE Preliminary catalog (Eisenhardt et al. 2019) provides new photometry and proper motion measurements using the original AllWISE processing techniques for data collected between 2010 and 2016, providing a factor of 10 improvement to the original AllWISE proper motion measurements, in addition to improving the depth and positional accuracy of sources as compared to AllWISE.

The proper motion accuracy in CatWISE is 10 mas yr $^{-1}$ for bright sources, 30 mas yr $^{-1}$ at $W 1 \approx 15.5 \mathrm{mag}$, and $100 \mathrm{mas} \mathrm{yr}^{-1}$ at $W 1 \approx 17$ mag, so an object must either be sufficiently bright or have a sufficiently high proper motion to perform this test. Two of our objects meet this criterion, EC 03103 and EC 05276, and their 
Table 2

Comparison of Gaia and CatWISE Proper Motion Measurements for Two Candidates in Our Sample

\begin{tabular}{lc}
\hline \hline EC 03103 & \\
\hline Gaia Source ID & 4720876181720327808 \\
Gaia DR2 $\mu_{\alpha} \cos (\delta)$ & $404.8 \pm 0.2$ \\
Gaia DR2 $\mu_{\delta}$ & $57.6 \pm 0.2$ \\
CatWISE Source Name & $\mathrm{J} 031122.06-621515.2$ \\
CatWISE $\mu_{\alpha} \cos (\delta)$ & $279.9 \pm 21.2$ \\
CatWISE $\mu_{\delta}$ & $80.3 \pm 19.3$ \\
\hline EC 05276 & \\
\hline Gaia Source ID & 4805782462481529600 \\
Gaia DR2 $\mu_{\alpha} \cos (\delta)$ & $-37.3 \pm 0.1$ \\
Gaia DR2 $\mu_{\delta}$ & $15.3 \pm 0.1$ \\
CatWISE Source Name & $\mathrm{J} 052912.09-430334.8$ \\
CatWISE $\mu_{\alpha} \cos (\delta)$ & $-397.3 \pm 34.4$ \\
CatWISE $\mu_{\delta}$ & $358.5 \pm 35.7$ \\
\hline
\end{tabular}

Note. All proper motion measurements are given in units of mas $\mathrm{yr}^{-1}$.

reported proper motions are given in Table 2. Both objects have discrepant proper motions in Gaia and CatWISE, consistent with their classification of having confused WISE photometry. Unfortunately, the sample size is not sufficient to evaluate the efficacy of this technique, but the two cases of confirmed source confusion demonstrate that it is a worthwhile check for large surveys of WISE infrared excesses.

\section{Conclusions}

Among the sample of 22 WISE-selected dusty white dwarf candidates, we find that eight are the result of source confusion, despite our attempts at vetting the sample prior to Spitzer observation. We show that ground-based, near-infrared imaging is insufficient for detecting the contaminants in our sample, but should still be employed when vetting candidates to rule out more obvious cases of source confusion. Astrometric filtering of candidates on the fainter end of the WISE catalog should also take into account the astrometric uncertainty, and we demonstrate the utility of filtering candidates using the FoM metric from the official GaiaAllWISE cross-match.

However, even when applying these techniques in combination one will fail to produce a clean sample of WISE-selected infrared excesses, and care must be taken when interpreting the statistical properties of WISE-selected infrared excesses. The fact remains that WISE-selected infrared excess candidates should be treated as guilty until proven innocent. The confusion limit is inherent to the WISE telescope and cannot be remedied by advanced processing. Future studies of $W I S E$-selected infrared excesses utilizing the new coadds and increased depth of the continued NEOWISE mission (Schlafly et al. 2019) could suffer from even higher contamination rates, as the survey depth is pushed further and further past the confusion limit.

The 14 confirmed excesses in our sample could also provide a nice increase to the known sample of dusty white dwarf stars, which currently stands between 40 and 50 systems (Farihi 2016). We emphasize that our confirmation does not signify their status as dusty white dwarf stars, as we cannot preclude the possibility of a brown dwarf companion as the source of the infrared excess. To-date, all confirmed dusty white dwarf stars have also shown signs of active accretion detectable as atmospheric metals, and the search for these is a necessary step for solidifying their infrared excess as circumstellar dust. Only 1 of the 14 Spitzer confirmed excesses in our sample has a literature detection of metals (EC 01071; Dennihy et al. 2017), and we are currently pursuing high resolution spectroscopic follow-up of the remaining candidates.

We would like to acknowledge Boris Gänsicke for comments and suggestions which improved this manuscript, and the anonymous referee for providing a swift and helpful report. This work is based in part on observations made with the Spitzer Space Telescope, which is operated by the Jet Propulsion Laboratory, California Institute of Technology under a contract with NASA. This research has made use of the NASA/ IPAC Infrared Science Archive, which is operated by the Jet Propulsion Laboratory, California Institute of Technology, under contract with the National Aeronautics and Space Administration. This publication makes use of data products from the Wide-field Infrared Survey Explorer, which is a joint project of the University of California, Los Angeles, and the Jet Propulsion Laboratory/California Institute of Technology, and NEOWISE, which is a project of the Jet Propulsion Laboratory/California Institute of Technology. WISE and NEOWISE are funded by the National Aeronautics and Space Administration. This work has made use of data from the European Space Agency (ESA) mission Gaia (https://www.cosmos.esa.int/gaia), processed by the Gaia Data Processing and Analysis Consortium (DPAC, https:// www.cosmos.esa.int/web/gaia/dpac/consortium). Funding for the DPAC has been provided by national institutions, in particular the institutions participating in the Gaia Multilateral Agreement.

Facilities: IRSA, Spitzer, WISE, Gaia.

Software: astropy (Astropy Collaboration et al. 2013).

\section{Appendix A Spectral Energy Distributions}

The spectral energy distributions of all 22 targets are shown here in Figure 4, following the style of Figure 2 

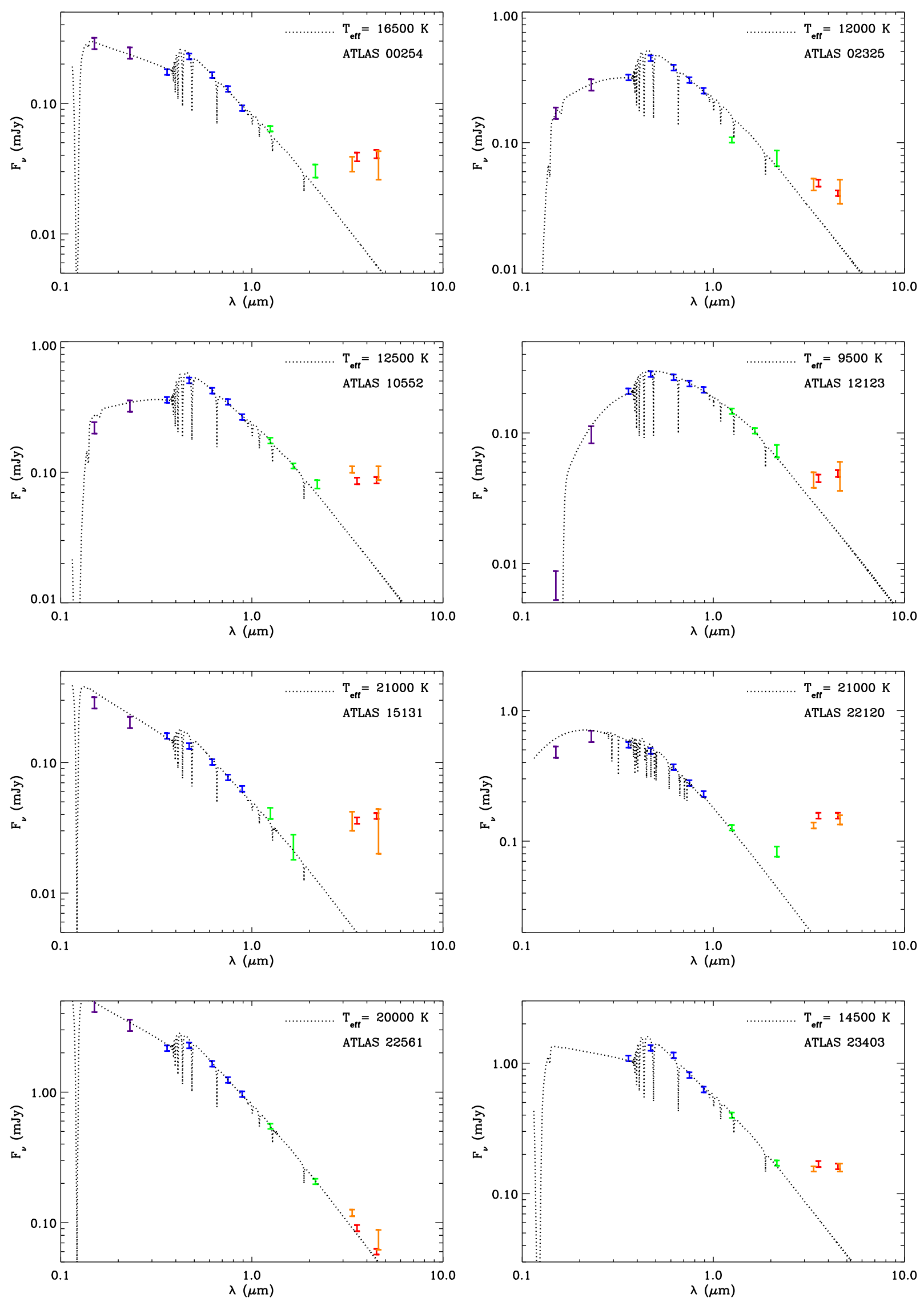

Figure 4. SEDs of remaining Spitzer targets. The cases where the Spitzer photometry (red) is consistent with the stellar models (dotted line) are targets with confused WISE (orange) excesses. 

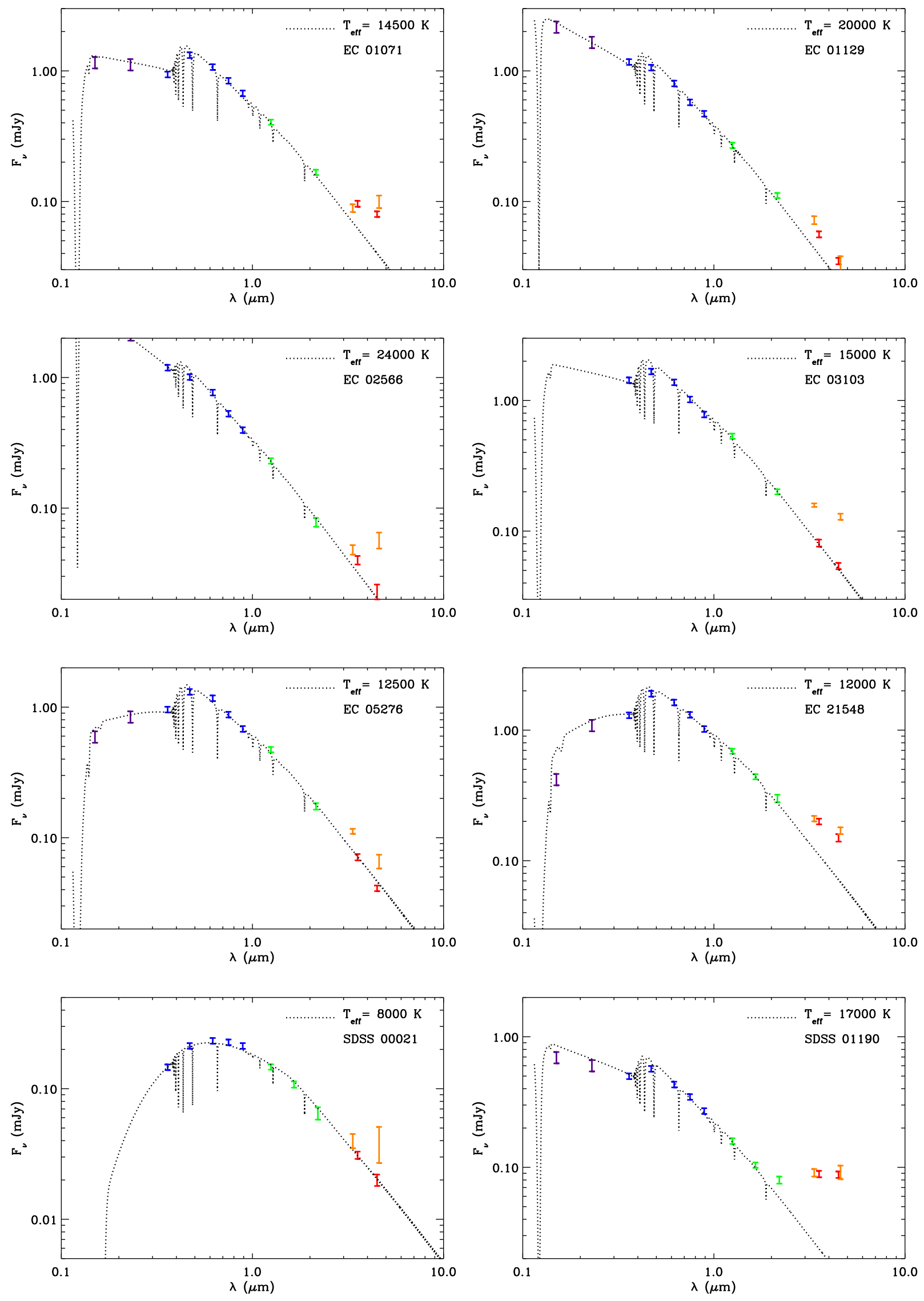

Figure 4. (Continued.) 

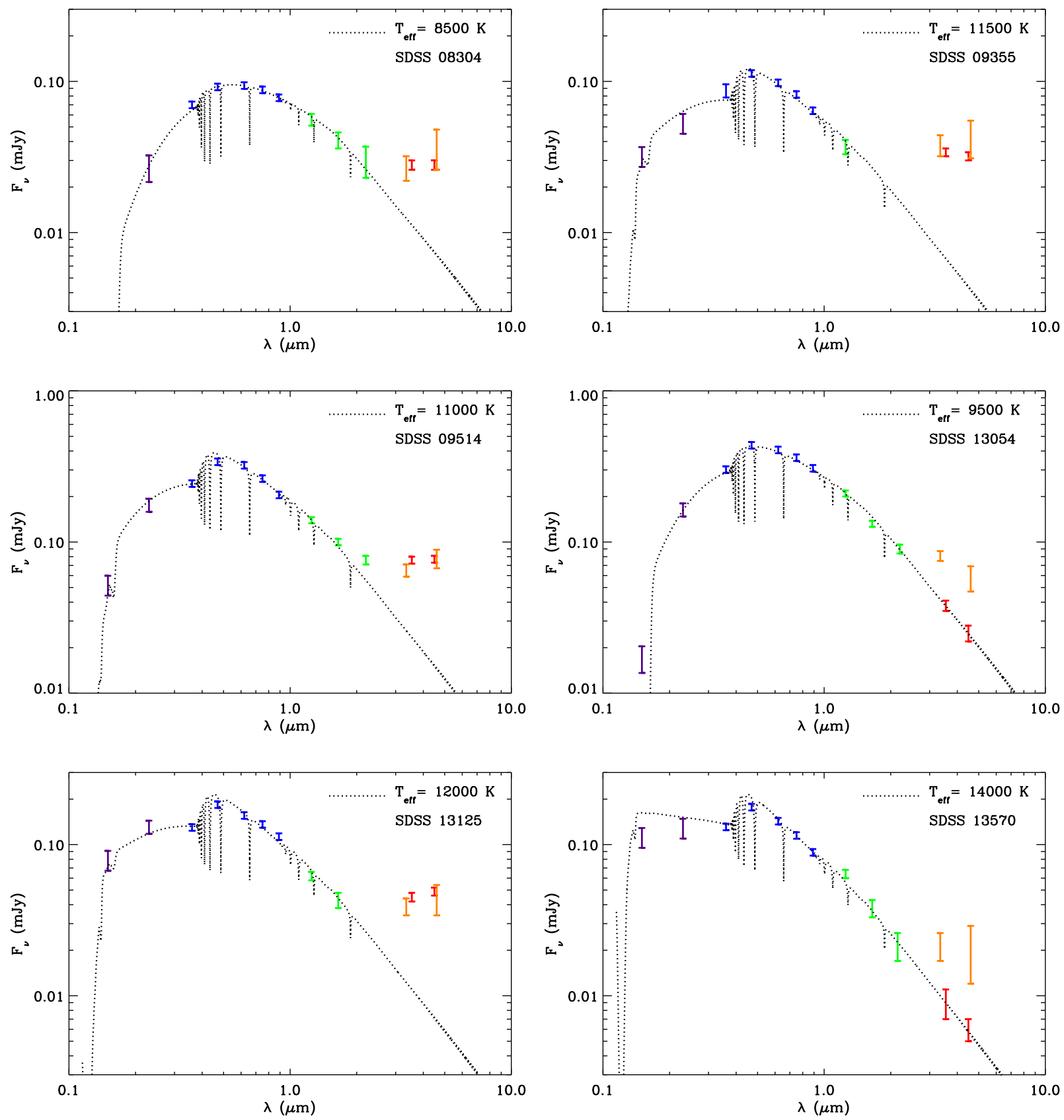

Figure 4. (Continued.)

\section{ORCID iDs}

Erik Dennihy 자 https://orcid.org/0000-0003-2852-268X Jay Farihi (i) https://orcid.org/0000-0003-1748-602X Nicola Pietro Gentile Fusillo (ㄷ) https://orcid.org/0000-00026428-4378

John H. Debes 주 https://orcid.org/0000-0002-1783-8817

\section{References}

Ahn, C. P., Alexandroff, R., Allende Prieto, C., et al. 2014, ApJS, 211, 17 Astropy Collaboration, Robitaille, T. P., Tollerud, E. J., et al. 2013, A\&A, 558, A33

Baraffe, I., Homeier, D., Allard, F., \& Chabrier, G. 2015, A\&A, 577, A42 Barber, S. D., Kilic, M., Brown, W. R., \& Gianninas, A. 2014, ApJ, 786, 77 Barber, S. D., Patterson, A. J., Kilic, M., et al. 2012, ApJ, 760, 26 Bayo, A., Rodrigo, C., Navascués, D., et al. 2008, A\&A, 492, 277
Binks, A. S., \& Jeffries, R. D. 2017, MNRAS, 469, 579

Chambers, K. C., Magnier, E. A., Metcalfe, N., et al. 2016, arXiv:1612.05560 Cotten, T. H., \& Song, I. 2016, ApJS, 225, 15

Cross, N. J. G., Collins, R. S., Mann, R. G., et al. 2012, A\&A, 548, A119

Cutri, R. M., Wright, E. L., \& Conrow, T. 2013a, Explanatory Supplement to the AllWISE Data Release Products, http://wise2.ipac.caltech.edu/docs/ release/allwise/expsup/index.html

Cutri, R. M., Wright, E. L., Conrow, T., et al. 2013b, yCat, 2328, 0

Debes, J. H., Hoard, D. W., Kilic, M., et al. 2011a, ApJ, 729, 4

Debes, J. H., Hoard, D. W., Wachter, S., Leisawitz, D. T., \& Cohen, M. 2011b, ApJS, 197, 38

Debes, J. H., Thévenot, M., Kuchner, M. J., et al. 2019, ApJL, 872, L25

Dennihy, E., Clemens, J. C., Debes, J. H., et al. 2017, ApJ, 849, 77

Dennihy, E., Debes, J. H., Dunlap, B. H., et al. 2016, ApJ, 831, 31

Eisenhardt, P. R. M., Marocco, F., Fowler, J. W., et al. 2019, The CatWISE Preliminary Catalog: Motions from WISE and NEOWISE Data, arXiv: 1908.08902

Farihi, J. 2016, NewAR, 71, 9

Farihi, J., Zuckerman, B., \& Becklin, E. E. 2008, ApJ, 674, 431 
Fazio, G. G., Hora, J. L., Allen, L. E., et al. 2004, ApJS, 154, 10

Gaia Collaboration, Brown, A. G. A., Vallenari, A., et al. 2018, A\&A, 616, A1

Gaia Collaboration, Prusti, T., de Bruijne, J. H. J., et al. 2016, A\&A, 595, A1

Gardner, J. P., Mather, J. C., Clampin, M., et al. 2006, SSRv, 123, 485

Gentile Fusillo, N. P., Gänsicke, B. T., \& Greiss, S. 2015, MNRAS, 448, 2260

Gentile Fusillo, N. P., Raddi, R., Gänsicke, B. T., et al. 2017, MNRAS, 469,621

Gentile Fusillo, N. P., Tremblay, P.-E., Gänsicke, B. T., et al. 2019, MNRAS, 482,4570

Girven, J., Gänsicke, B. T., Steeghs, D., \& Koester, D. 2011, MNRAS, 417,1210

Hambly, N. C., Collins, R. S., Cross, N. J. G., et al. 2008, MNRAS, 384, 637

Hoard, D. W., Debes, J. H., Wachter, S., Leisawitz, D. T., \& Cohen, M. 2013, ApJ, 770, 21

Irwin, M. J., Lewis, J., Hodgkin, S., et al. 2004, Proc. SPIE, 5493, 411

Kennedy, G. M., \& Wyatt, M. C. 2012, MNRAS, 426, 91

Koester, D. 2010, MmSAI, 81, 921

Lawrence, A., Warren, S. J., Almaini, O., et al. 2007, MNRAS, 379, 1599

Mainzer, A., Bauer, J., Cutri, R. M., et al. 2014, ApJ, 792, 30

Makovoz, D., Roby, T., Khan, I., \& Booth, H. 2006, Proc. SPIE, 6274, 62740C
Marrese, P. M., Marinoni, S., Fabrizio, M., \& Altavilla, G. 2019, A\&A, 621, A144

Marrese, P. M., Marinoni, S., Fabrizio, M., \& Giuffrida, G. 2017, A\&A, 607, A105

Martin, D. C., Fanson, J., Schiminovich, D., et al. 2005, ApJL, 619, L1

McMahon, R. G., Banerji, M., Gonzalez, E., et al. 2013, Msngr, 154, 35

Patel, R. I., Metchev, S. A., \& Heinze, A. 2014, ApJS, 212, 10

Patel, R. I., Metchev, S. A., Heinze, A., \& Trollo, J. 2017, AJ, 153, 54

Rebassa-Mansergas, A., Solano, E., Xu, S., et al. 2019, MNRAS, 489, 3990

Schlafly, E. F., Meisner, A. M., \& Green, G. M. 2019, ApJS, 240, 30

Shanks, T., Metcalfe, N., Chehade, B., et al. 2015, MNRAS, 451, 4238

Silverberg, S. M., Kuchner, M. J., Wisniewski, J. P., et al. 2018, ApJ, 868, 43

Skrutskie, M. F., Cutri, R. M., Stiening, R., et al. 2006, AJ, 131, 1163

Stobie, R. S., Kilkenny, D., O’Donoghue, D., et al. 1997, MNRAS, 287, 848

Werner, M. W., Roellig, T. L., Low, F. J., et al. 2004, ApJS, 154, 1

Wilson, T. G., Farihi, J., Gänsicke, B. T., \& Swan, A. 2019, MNRAS, 487, 133

Wilson, T. J., \& Naylor, T. 2017, MNRAS, 468, 2517

Wilson, T. J., \& Naylor, T. 2018, MNRAS, 481, 2148

Wolf, C., Onken, C. A., Luvaul, L. C., et al. 2018, PASA, 35, e010

Wright, E. L., Eisenhardt, P. R. M., Mainzer, A. K., et al. 2010, AJ, 140, 1868 